\title{
Evaluation of neck node response after radiotherapy: minimizing equivocal results
}

\author{
Remco de Bree $^{1}$ - Otto S. Hoekstra ${ }^{2}$
}

Published online: 6 January 2016

(C) Springer-Verlag Berlin Heidelberg 2016

\begin{abstract}
About two-thirds of patients with head and neck squamous cell carcinoma (HNSCC) originating from the mucosal linings of the upper aerodigestive tract present with advanced stage disease. If the primary tumour is located in the pharynx or larynx, patients are generally treated with radiotherapy with or without chemotherapy. In patients with functionally unresectable HNSCC (resectable but high morbidity of surgical treatment expected) who are treated with this nonsurgical treatment, salvage surgery is kept in reserve in case residual disease occurs.

The decision to perform a neck dissection following (chemo)radiotherapy is clear when patients have proven residual nodal disease in the neck. However, distinguishing between residual metastasis and radiotherapy sequelae is difficult in most patients with a residual neck mass, since posttreatment induration and fibrosis make accurate clinical assessment difficult. Because no reliable clinical parameters are available to predict pathological neck status after (chemo)radiotherapy, routine planned neck dissection is performed in many institutions. The integration of planned neck dissection into the multidisciplinary management of patients with locoregionally advanced head and neck cancer treated with
\end{abstract}

This Editorial Commentary refers to the article http://dx.doi.org/10.1007/ s00259-015-3194-3

Remco de Bree

r.debree@umcutrecht.nl

1 Department of Head and Neck Surgical Oncology, UMC Utrecht Cancer Center, University Medical Center Utrecht, P.O. Box 85500, 3508 GA Utrecht, The Netherlands

2 Department of Radiology and Nuclear Medicine, VU University Medical Center, Amsterdam, The Netherlands concomitant chemoradiotherapy is highly effective in controlling residual neck nodal disease. However, in the majority of neck dissection specimens, no viable tumour cells are found. Moreover, neck dissection after radiotherapy is associated with a significant risk of wound healing problems and shoulder morbidity. On the other hand, late recurrences in the neck are rarely surgically salvageable and are associated with an increased risk of metastatic spread to distant sites. Therefore, early detection of residual neck disease after (chemo)radiotherapy is important [1-4].

There is a tendency to perform neck dissections after (chemo)radiotherapy only if indicated by posttreatment diagnostic evaluation of the neck (physical examination, imaging and/or cytological evaluation) [1-4]. Van der Putten et al. [5] reported on 129 patients with neck recurrence out of 540 HNSCC patients who underwent neck dissection after chemoradiotherapy because of clinical suspicion of neck recurrence. In this series a planned neck dissection would have been unnecessary in $76 \%$ of the patients with $\mathrm{N} 2 / \mathrm{N} 3$ disease. In patients with $\mathrm{N} 0 / \mathrm{N} 1$ neck the number of unnecessary neck dissections would be even higher (92.8\%) [5].

For more individualized salvage neck treatment after (chemo)radiotherapy, the head and neck surgeon needs a reliable selection tool with clear results. For improved survival, high sensitivity is needed: residual neck disease should be detected early. Delaying a neck dissection allows more time for both cancer progression and radiation-induced fibrosis, which may hamper the feasibility of a neck dissection and increase surgical complications. To avoid unnecessary salvage neck dissections, a high negative predictive value is necessary. Since the head and neck surgeon only wants to know if a salvage neck dissection should be performed, equivocal test results are not useful.

Although different imaging modalities have been investigated [6], ${ }^{18} \mathrm{~F}-\mathrm{FDG}$ PET/CT seems to be the most promising 
diagnostic technique for response assessment following (chemo)radiotherapy. Unfortunately, it is not yet established how these PET/CT scans should be performed and interpreted for optimal use in clinical practice. This is illustrated by the wide variation in sensitivity $(25-100 \%)$ and negative predictive value $(14-100 \%)[7,8]$. It must be emphasized that because of the low incidence (less than $10 \%$ ) of residual disease due to improved (chemo)radiotherapy, high negative predictive values can be obtained relatively easily nowadays. The reported apparent variability of the operational characteristics of ${ }^{18}$ F-FDG PET in this setting may be related to, for example, the included patient spectrum, the timing of PET after therapy, PET positivity criteria, and the definition of the reference test (e.g. methods and duration of follow-up).

PET imaging obtained too soon after radiotherapy has been associated with a high rate of false-positive findings due to postradiotherapy soft tissue effects, and false-negative findings because of the residual viable cancer cells not having sufficient time to repopulate to a level that can be detected by PET. If the time interval between the end of therapy and PET scanning increases the negative predictive value improves. In a meta-analysis of the diagnostic performance of posttreatment ${ }^{18} \mathrm{~F}$-FDG PET imaging in head and neck cancer, Gupta et al. [9] found that the sensitivity of PET scans performed 12 or more weeks after radiotherapy was significantly higher than that of scans performed within 12 weeks. Recently, Leung et al. [10] sought to determine the optimal timing of the first posttreatment ${ }^{18}$ F-FDG PET scan after chemoradiotherapy and found that PET/CT scans obtained within 7 weeks after treatment were less accurate than scans obtained at later time points. Furthermore, the accuracy of these scans did not differ significantly whether they were obtained between 7 and 10 weeks, between 11 and 14 weeks, or after 15 weeks or more [10]. The optimal timing is still a matter of debate, particularly because of the better chance of salvage when residual disease is diagnosed in a timely manner. An interval between 8 and 12 weeks after the end of treatment is generally accepted.

${ }^{18}$ F-FDG uptake can be evaluated quantitatively and qualitatively. Qualitative assessment of the metabolic rate of glucose by dynamic scanning to obtain the rate of glucose consumption is not feasible in daily clinical practice. Semiquantitative assessments include standardized uptake values (SUV), of which maximum SUV (SUVmax) is most often used, metabolic tumour volume and total lesion glycolysis. Visual assessment is commonly used, but standardized criteria are lacking. Different Likert scales have been investigated. As can be done with continuous data (e.g. SUV), such ordinal scales enable determination of the best dichotomization, e.g. considering equivocal results positive or negative. ROC curves seam be useful for determining the optimal cutoff values, but sensitivity and specificity are not equally important in this clinical problem. An unnecessary neck dissection outweighs missing residual disease which potentially compromises survival. The same holds true for finding a significant difference for each criterion within a scoring system. It is only a prerequisite for further determination of the best dichotomized categorization for use in daily clinical practice.

Several SUVmax cut-off values ranging from 2.0 to 3.0 have been reported [11-15]. Each of these cut-off values was considered optimal in the series in which it was reported. Besides patient population, acquisition protocol, definition of region of interest and the reconstruction algorithm used in the different institutions may affect the choice of the best cut-off value to detect residual neck disease after (chemo)radiotherapy. Guidelines for standardization of PET imaging and quantification of ${ }^{18} \mathrm{~F}$-FDG uptake have been developed [16]. However, too often publications fail to provide sufficient details on the imaging methodology to assess whether quantitative data can be compared with the results of other studies. Finally, any data-driven threshold for test positivity requires prospective external validation. The obvious potential added value of quantification (e.g. by avoiding observer variation) can only be exploited and capitalized upon after standardization of the methodology of the technique.

For qualitative PET interpretation several Likert scores have been used. Sjövall et al. [17] used the five-point Deauville scale as applied in malignant lymphoma (relating residual uptake to mediastinal blood pool and liver uptake). Marcus et al. [18] recently proposed the five-point "Hopkins scale" based on intensity (similar to the Deauville scale, but using the jugular vein rather than the mediastinal blood pool), adding a description of aspects of residual uptake (focal or diffuse). Krabbe et al. [19] graded the confidence of image interpretation using a five-point scale $(0$ definitely no tumour, 1 probably no tumour, 2 equivocal, 3 probably tumour, and 4 definite tumour). Schouten et al. [20] used a similar system based on aspect and intensity. The different qualitative scoring systems are thus based on focal uptake assessed in relation to adjacent tissue, internal jugular vein, mediastinum or liver uptake [17, 18, 21].

Baseline PET imaging may be useful in PET reading for evaluating treatment response [22]. Another option for improving response evaluation might be to repeat PET scans during follow-up. Although in the study by Sjövall et al. [17] this seemed not to improve the response evaluation, in the study by Krabbe et al. [19] the detection of residual neck disease improved. Preliminary data [20] have indicated that a combination of ${ }^{18} \mathrm{~F}$-FDG PET and DW-MRI may be an attractive option for optimizing the decision making to perform a neck dissection following chemoradiotherapy. Sjövall et al. [17] conclude that a Likert scale seems to satisfy the 
requirements for a common qualitative way of assessing PET scans and also the reporting of PET results in the evaluation of head and neck cancer. Consensus regarding qualitative assessment would facilitate PET reporting in clinical practice and enable comparative studies between patients and institutions.

For malignant lymphoma the Deauville five-point scoring system was developed. It was subsequently compared with other interpretation systems and found to be accurate and reproducible. It was validated in an international multicentre study and was accepted as a standard reporting criterion in clinical practice and for clinical trials [23, 24]. Sjövall et al. [17] made the first step in this process of improving response evaluation in head and neck cancer by comparing different interpretation systems. However, larger series preferably in multicentre collaborative studies are needed to compare different test positivity criteria, and to be able to comprehensively study the potential impact of other variables (e.g. timing, tumour subtype, reference standards etc.), and ultimately to validate the proposed taxonomy.

\section{Compliance with ethical standards}

Conflicts of interest None.

\section{References}

1. Narayan K, Crane CH, Kleid S, Hughes PG, Peters LJ. Planned neck dissection as an adjunct to the management of patients with advanced neck disease treated with definitive radiotherapy: for some or for all? Head Neck. 1999;21:606-13.

2. Corry J, Smith JG, Peters LJ. The concept of a planned neck dissection is obsolete. Cancer J. 2001;7:472-4.

3. Grabenbauer GG, Rodel C, Ernst-Stecken A, Brunner T, Hornung $\mathrm{J}$, Kittel K, et al. Neck dissection following radiochemotherapy of advanced head and neck cancer - for selected cases only? Radiother Oncol. 2003;66:57-63.

4. Pellitteri PK, Ferlito A, Rinaldo A, Shah JP, Weber RS, Lowry J, et al. Planned neck dissection following chemoradiotherapy for advanced head and neck cancer: is it necessary for all? Head Neck. 2006;28:166-75.

5. van der Putten L, van den Broek GB, de Bree R, van den Brekel MW, Balm AJ, Hoebers FJ, et al. Effectiveness of salvage selective and modified radical neck dissection for regional pathological lymphadenopathy after chemoradiation. Head Neck. 2009;31: 593-603.

6. Ojiri H, Mancuso AA, Mendenhall WM, Stringer SP. Lymph nodes of patients with regional metastases from head and neck squamous cell carcinoma as a predictor of pathologic outcome: size changes at $\mathrm{CT}$ before and after radiation therapy. AJNR Am J Neuroradiol. 2002;23:1627-31.

7. Isles MG, McConkey C, Mehanna HM. A systematic review and meta-analysis of the role of positron emission tomography in the follow up of head and neck squamous cell carcinoma following radiotherapy or chemoradiotherapy. Clin Otolaryngol. 2008;33: 210-22.
8. de Bree R, van der Putten L, Brouwer J, Castelijns JA, Hoekstra OS, Leemans CR. Detection of locoregional recurrent head and neck cancer after (chemo)radiotherapy using modern imaging. Oral Oncol. 2009;45:386-93.

9. Gupta T, Master Z, Kannan S, Agarwal JP, Ghsoh-Laskar S, Rangarajan V. Diagnostic performance of post-treatment FDG PET or FDG PET/CT imaging in head and neck cancer: a systematic review and meta-analysis. Eur J Nucl Med Mol Imaging. 2011;38(11):2083-95.

10. Leung AS, Rath TJ, Hughes MA, Kim S, Branstetter BF 4th. Optimal timing of first posttreatment FDG PET/CT in head and neck squamous cell carcinoma. Head Neck. 2015. doi: 10.1002/ hed.24112.

11. Yao M, Graham MM, Hoffman HT, Smith RB, Funk GF, Graham $\mathrm{SM}$, et al. The role of post-radiation therapy FDG PET in prediction of necessity for post-radiation therapy neck dissection in locally advanced head-and-neck squamous cell carcinoma. Int J Radiat Oncol Biol Phys. 2004;59(4):1001-10.

12. Nam SY, Lee SW, Im KC, Kim JS, Kim SY, Choi SH, et al. Early evaluation of the response to radiotherapy of patients with squamous cell carcinoma of the head and neck using 18FDG-PET. Oral Oncol. 2005;41(4):390-5.

13. Moeller BJ, Rana V, Cannon BA, Williams MD, Sturgis EM, Ginsberg LE, et al. Prospective risk-adjusted [18F]Fluorodeoxyglucose positron emission tomography and computed tomography assessment of radiation response in head and neck cancer. J Clin Oncol. 2009;27:2509-15.

14. Gourin CG, Boyce BJ, Williams HT, Herdman AV, Bilodeau PA, Coleman TA. Revisiting the role of positron-emission tomography/ computed tomography in determining the need for planned neck dissection following chemoradiation for advanced head and neck cancer. Laryngoscope. 2009;119(11):2150-5.

15. Zundel MT, Michel MA, Schultz CJ, Maheshwari M, Wong SJ, Campbell $\mathrm{BH}$, et al. Comparison of physical examination and fluorodeoxyglucose positron emission tomography/computed tomography 4-6 months after radiotherapy to assess residual headand-neck cancer. Int J Radiat Oncol Biol Phys. 2011;81(5):e825-32.

16. Boellaard R, Delgado-Bolton R, Oyen WJ, Giammarile F, Tatsch $\mathrm{K}$, Eschner W, et al. FDG PET/CT: EANM procedure guidelines for tumour imaging: version 2.0. Eur J Nucl Med Mol Imaging. 2015;42(2):328-54.

17. Sjövall J, Bitzén U, Kjellén E, Nilsson P, Wahlberg P, Brun E. Quantitative interpretation of positron emission tomography scans using a Likert scale to assess neck node response to radiotherapy in head and neck cancer. Eur J Nucl Med Mol Imaging. 2015. doi:10. 1007/s00259-015-3194-3

18. Marcus C, Ciarallo A, Tahari AK, Mena E, Koch W, Wahl RL, et al. Head and neck PET/CT: therapy response interpretation criteria (Hopkins criteria) - interreader reliability, accuracy, and survival outcomes. J Nucl Med. 2014;55(9):1411-6.

19. Krabbe CA, Pruim J, Dijkstra PU, Balink H, van der Laan BF, de Visscher JG, et al. 18F-FDG PET as a routine posttreatment surveillance tool in oral and oropharyngeal squamous cell carcinoma: a prospective study. J Nucl Med. 2009;50(12):1940-7.

20. Schouten CS, de Graaf P, Alberts FM, Hoekstra OS, Comans EF, Bloemena E, et al. Response evaluation after chemoradiotherapy for advanced nodal disease in head and neck cancer using diffusionweighted MRI and 18F-FDG-PET-CT. Oral Oncol. 2015;51(5): 541-7.

21. Porceddu SV, Pryor DI, Burmeister E, Burmeister BH, Poulsen MG, Foote MC, et al. Results of a prospective study of positron emission tomography-directed management of residual nodal abnormalities in node-positive head and neck cancer after definitive radiotherapy with or without systemic therapy. Head Neck. 2011;33(12):1675-82. 
22. Wahl RL, Jacene H, Kasamon Y, Lodge MA. From RECIST to PERCIST: evolving considerations for PET response criteria in solid tumours. J Nucl Med. 2009;50 Suppl 1:122S-50S.

23. Biggi A, Gallamini A, Chauvie S, Hutchings M, Kostakoglu L, Gregianin $\mathrm{M}$, et al. International validation study for interim PET in ABVD-treated, advanced-stage Hodgkin lymphoma: interpretation criteria and concordance rate among reviewers. J Nucl Med. 2013;54(5):683-90.

24. Kajáry K, Molnár Z, Györke T, Szakáll Jr S, Molnár P, Lengyel Z. Comparison of the International Harmonization Project, London and Gallamini criteria in the interpretation of $18 \mathrm{~F}-\mathrm{FDG}$ PET/CT examinations after first-line treatment in Hodgkin's lymphoma. Nucl Med Commun. 2014;35(2):169-75. 